\title{
Fucoidans as a Potential Nutraceutical in Combating Atherosclerotic Cardiovascular Diseases
}

\author{
Weslley F Braga*, Edenil C Aguilar and Jacqueline I Alvarez-Leite \\ Department of Biochemistry and Immunology, Brazil
}

*Corresponding author: Jacqueline I Alvarez-Leite, Department of Biochemistry and Immunology, Caixa Postal 486 -

30161-970 - Belo Horizonte, MG Brazil

\begin{abstract}
ARTICLE INFO
Received: 幽 September 19, 2019

Published: 彗 September 24, 2019

Citation: Weslley F Braga, Edenil C A, Jacqueline I Alvarez-Leite. Fucoidans as a Potential Nutraceutical in Combating Atherosclerotic Cardiovascular Diseases. Biomed J Sci \& Tech Res 21(3)-2019. BJSTR. MS.ID.003615.
\end{abstract}

Keywords: Fucoidan, Nutraceutical, Atherosclerosis, Inflammation

\begin{abstract}
The base of atherosclerotic cardiovascular diseases (ACVD) is the triad dyslipidemia, inflammation and thrombosis. Fucoidans are sulfated polysaccharides components from brown algae Phaeophyceae class related to several biological activities. In this article, we discuss some "in vitro" and experimental studies describing actions of fucoidan that could be beneficial in controlling ACVD. Nonetheless, clinical studies are still scarce in the literature. It has been shown that fucoidans reduce blood lipids, increase LDL receptor and reduce scavenger receptors, impair migration and activation of immune cells and cytokine production and also reduce platelet aggregation and increase fibrinolysis. Fucoidan is a potential nutraceutical that could be useful as an adjuvant in preventing or treating ACVD. Nonetheless, future clinical studies are needed to confirm these effects in humans.
\end{abstract}

Abbreviations: AP-1: Activator Protein-1; ACVD: Atherosclerotic Cardiovascular Diseases; JNK: C-Jun N-Terminal Kinase; Erk1/2: Extracellular Signal-Regulated Kinase1/2; HDL: High-Density Lipoprotein; HO-1: Heme Oxygenase 1; HMG-CoA: Hydroxy-Methyl-Glutaryl CoA; NOS: Inducible Nitric Oxide Synthase; IFN- $\gamma$ : InterferonGamma; IL: Interleukin; LPL: Lipoprotein lipase; MMP: Matrix Metalloproteinases; MAPK: Mitogen-Activated Protein Kinase MCP1: Monocyte Chemotactic Protein-1 MCP1-CCL2; NO: Nitric oxide; NF- $\kappa$ B: Nuclear factor kappa B; OxLDL: Oxidized LDL; ROS: Reactive Oxygen Species; LDL: Reduces Low-Density Lipoprotein; SOD1: Superoxide Dismutase 1; tPA: Tissue Plasminogen Activator; TNF: Tumor Necrosis Factor

\section{Introduction}

Atherosclerotic cardiovascular diseases (ACVD) are one of the most relevant world health problems, accounting for more than $30 \%$ of all global deaths [1]. It englobes primary acute myocardial infarction, but also ischemic stroke, intermittent claudication due to obstruction of peripherical arteries and aorta aneurysm [2]. The base of ACVD is the triad dyslipidemia, inflammation, and thrombosis. High levels of low-density lipoprotein (LDL) contributes to endothelial dysfunction and inflammation, culminating in atherothrombosis, blood flow obstruction and tissue ischemia/ necrosis. Several risk factors, including diabetes, smoking, sedentary lifestyle, familial history, obesity and hypertension may induce or accelerate endothelial injury, contributing to the progression of atherosclerosis [2].
LDL is the primary causal factor associated with atherosclerosis. In the early phase of atherogenesis, the excess of blood LDL passes through the dysfunctional endothelium and remains in the intimal layer or forms aggregates with proteoglycans. Abnormal LDL, mainly oxidized or glycated (oxLDL) can also promote or accelerate this process [3]. Endothelial activation by oxLDL is characterized by the higher expression of adhesion molecules, secretion of inflammatory mediators and leukocyte recruitment, mainly monocytes, to the lesion site [4]. In the arterial intima layer, recruited monocytes differentiate into macrophages and uptake oxLDL by scavenger receptors (such as CD36 and SRA), transforming into foam cell, the primary atherosclerotic lesion. LDL receptor related protein (LRP) in the surface of smooth muscle cells (SMC) (migrated from media 
layer) can bind LDL-proteoglycan aggregates, also generating foam cells. The continuous and unregulated oxLDL endocytosis will lead to the expansion of foam cells, forming the atheroma. As the plaque progresses, new events contribute to the lipid-rich necrotic core formation such as local hypoxia, intraplaque angiogenesis and thrombosis, $\mathrm{T}$ and $\mathrm{B}$ cell migration, cytokine production and deposition of apoptotic debris with inefficient efferocytosis [5]. Lesions are covered by a fibrous cap formed by the extracellular matrix produced by smooth muscle cells that stabilize the atheroma [6]. Nonetheless, the production of matrix metalloproteinases (mainly MMP-2 and MMP-9) by macrophage and the inflammatory mediators (such as interferon-gamma) released by $\mathrm{T}$ cells may degrade or inhibit the production of the extracellular matrix, causing plaque vulnerability with subsequent rupture [7].

Plaque rupture propitiates the contact of thrombogenic components (such as tissue factor) and other intraplaque material with blood, leading to thrombus formation, arterial obstruction, and ischemia of the tissue drained by that artery. Despite the increasing number of drugs to prevent and treat atherosclerosis, diet still integrates the first-line treatment [8]. Moreover, phytotherapeuticagents and nutraceuticals could be used as adjuvant therapy, permitting a safer and longer use compared to several drugs [9]. In the context of atherosclerosis, fucoidans could be an exciting therapeutic adjuvant due to several actions that can interfere with atherosclerosis progression $[10,11]$. Here we will discuss the main biological activities of fucoidan that make it a potential nutraceutical helping the management of ACVD.

\section{General Characteristics of Fucoidans}

Oceans and seas are sources of food for the whole world population due to the abundance of biological and natural resources. The diversity of marine species of fish, mollusks, crustaceans, and others offers multiple nutrients sources for a healthy diet. Seaweeds are, in many countries, a source of dietary nutrients and also a nutraceutical agent. Fucoidans are algae components that have been studied and related to several biological activities. Fucoidan is one of several marine sulfated polysaccharides, called "fucans" and mainly found in cell walls and the intercellular spaces of the brown algae from Phaeophyceae class [12].

The composition of fucoidans varies according to algae species, geographic location, harvesting season, climate and the extraction method [13]. Fucoidan is obtained from different species of brown algae and designates a group of fucose-containing sulfated polysaccharides. The fucoidans present similar structures: a backbone built of $(1 \rightarrow 3)$-linked $\alpha$-L-fucopyranosyl or a structure alternating $(1 \rightarrow 3)$ and $(1 \rightarrow 4)$-linked $\alpha$-L-fucopyranosyl residues. Nonetheless, it includes sulfated galactofucans with backbones built of $(1 \rightarrow 6)$ - $\beta D$-galacto- or $(1 \rightarrow 2)-\beta$-D-mannopyranosyl units with fucose or fuco-oligosaccharide branching or glucuronic acid, xylose or glucose substitutions [14].
The characteristics of fucoidan are also influenced by the purification processes. The lack of a standardized purification procedure leads to differences in the structure of the extracted polysaccharide. Despite these structural differences, fucoidan has been used as a nutraceutical supplement in several countries. The biological properties of fucoidans are determined by characteristics, such as molecular weight, structure, frequency, the position of sulfate groups, and the organization of sulfated domains [15]. Several "in vitro" and "in vivo" studies have described the fucoidan activities which could influence atherosclerosis development. Although studies of the biological activities of fucoidans have been conducted using molecules from different algae, we are presenting their origins and related activities in Table 1 and name all, regardless of the origin as fucoidan [16-38] (Table 1).

Certain portions of polysulfide-chain fucoidan mimic natural ligand-protein receptors to glycosaminoglycan, including heparan sulfate [39-41]. It is known that the binding of glycosaminoglycan to various tissue proteins leads to post-traditional modifications, which determine cell migration, proliferation, differentiation, as well as inflammation and angiogenesis [42]. Consequently, glycosaminoglycan mimics, such as fucoidans can modulate cell signaling [43].

Table 1: Biological activities of fucoidans from different species of brown seaweeds.

\begin{tabular}{|c|c|}
\hline Activity & Seaweed \\
\hline Hypolipidemic [16-20] & $\begin{array}{c}\text { Fucus vesiculosus; Japonica } \\
\text { laminaria; Cladosiphon cladosiphon; }\end{array}$ \\
\hline Antioxidant [21-25] & $\begin{array}{c}\text { Fucus vesiculosus; Japonica } \\
\text { laminaria; Ascophyllum nodosum }\end{array}$ \\
\hline Anti-inflammatory [26-30] & $\begin{array}{c}\text { Fucus vesiculosus; Ecklonia cava; } \\
\text { Sargasum horneri }\end{array}$ \\
\hline $\begin{array}{c}\text { Antiadipogenic and Obesity } \\
\text { [31-34] }\end{array}$ & $\begin{array}{c}\text { Undaria pinnatifida; Fucus } \\
\text { vesiculosus }\end{array}$ \\
\hline Anticoagulant and & $\begin{array}{c}\text { Fucus vesiculosus; Fucus } \\
\text { evanescens; Canistrocarpus } \\
\text { cervicornis }\end{array}$ \\
\hline Cardioprotective [19,38] & $\begin{array}{c}\text { Cladosiphon cladosiphon; Japonica } \\
\text { Laminaria }\end{array}$ \\
\hline
\end{tabular}

\section{Fucoidan as an Antithrombotic Agent}

Heparin is already used as an antithrombotic and anticoagulant agent. Due to the similarity with heparin, several fucoidans were tested for their abilities to stimulate fibrinolytic and anti-coagulant molecules [44]. In a murine model of thrombosis, fucoidan was showed an antithrombotic effect stronger than heparin [27]. When mechanisms were explored, it was found that the effects of fucoidan on the factor Xa and thrombin were weaker compared with heparin. These results suggest that the effects of fucoidan is due to the binding with heparin cofactor II and not with antithrombin, as occurs with heparin [27]. Moreover, unlike heparin, fucoidan stimulates tissue Plasminogen Activator (tPA) and induced fibrinolysis by protecting plasmin activity from $\alpha 2$-plasmin inhibitor and decreased fibrin 
polymer formation [45]. It was also demonstrated that fucoidans with different molecular weight possess diverse antithrombotic activities [46]. The anti-thrombotic activity seems to occur only with fucoidans with a minimum load density of 0.5 sulfate groups per sugar unit and a size of at least 70 sugar units [47].

\section{Fucoidan as a Hypolipidemic and Antiatherogenic Agent}

Hyperlipidemia is associated with increased risk of developing CVD diseases. Mice fed a high-fat diet (HFD) and receiving fucoidan (via gavage) presented lower levels of total cholesterol (TC), triglycerides (TG) and low-density lipoprotein cholesterol (LDL-C) while high-density lipoprotein cholesterol (HDL-C) concentration was increased [18]. The mechanism was elucidated in adipocytes cultivated in the presence of fucoidan from Fucus vesiculosus [16]. Fucoidan increased lipoprotein lipase (LPL) and apolipoprotein C-II (Apo C-II) secretion from adipocytes. These effects are similar to those seen with heparin, suggesting the same mechanism for both. Fucoidan can modulate cellular uptake and synthesis of cholesterol and fatty acids [17]. It was demonstrated that fucoidan attenuated the expression of enzymes involved in lipogenesis (fatty acid synthase, acetyl-CoA carboxylase), cholesterogenesis (HMG-CoA reductase) and increase lipoprotein uptake (LDL receptor) through the modulation of SREBP-2 [17]. In a model of isoproterenolinduced myocardial infarction, fucoidan administration reduced hypertriglyceridemia and hypercholesterolemia by reducing LDL-C and increasing HDL-C concentrations [19]. Fucoidan was also related to lower levels of lipid peroxidation, cardiac enzymes release and myocardial damage [19]. Moreover, a murine model of atherosclerosis (ApoE ${ }^{\text {shl}}$ ) fed on HFD supplemented with 1\% or $5 \%$ of fucoidan showed reduced hepatic steatosis, as well as lesser atherosclerotic lesion extension, lipid peroxidation and macrophage infiltration $[20,31]$.

\section{Fucoidan as an Antioxidant Agent}

Numerous in vitro and in vivo assays have evaluated the antioxidant efficacy of fucoidan. It was demonstrated that fucoidan eliminates hypochlorous acid superoxide radicals and prevented superoxide radicals, hydroxyl radicals, and lipid peroxide formation $[23,37,48-50]$. The antioxidant activity, like antithrombotic activity, is dependent on its molecular weight and sulfate content. The low molecular weight fractions had a better inhibitory effect on $\mathrm{Cu}^{2+}$ induced LDL oxidation compared to high molecular weight ones [28]. In an "in vivo" study using LDL receptor knockout (LDLR-/) mice, the oxLDL receptor (LOX-1) and reactive oxygen species (ROS) related protein were negatively regulated in the aorta after fucoidan supplementation [51]. These results suggest that fucoidan reduces oxidative stress and atherogenesis in animal models. Similar results were obtained in streptozotocin-induced diabetic mice that presented a lower ROS production in aorta smooth muscle cells after fucoidan treatment [52]. The mechanism may be linked to the inhibition of the Mitogen Activated Protein kinases (MAPK) and Nrf2/ERK signaling that mediates the expressions of HO-1 (heme oxygenase 1) and SOD1 (superoxide dismutase 1) [53,54].

\section{Fucoidan as an Anti-Inflammatory Agent.}

Fucoidans are potent inhibitors of leukocyte migration and platelet activation due to their interaction with $\mathrm{L}$ and P-selectins, acting as a "selectin blocker" [55]. P-selectin is found in the surface of activated platelets as well as activated endothelium [56]. The adhesion of leukocytes (neutrophils and monocytes) to the activated vascular endothelium and the adhesion of leukocytes to activated platelets are facilitated by selectins [57]. Fucoidan probably acts similarly to heparan sulfate, presenting a spatial structure of sulfated saccharide that is similar to the fucosylated, sialylated, or sulfated oligosaccharides present in the cell surface [13]. Fucoidans also suppress the activity of several inflammatory mediators. The crucial action is the inhibition of nuclear factor-kappa B (NF-кB) signaling. NF- $\mathrm{KB}$ is an important transcription factor involved in the inflammatory response. Once activated by different stimuli, NF-KB is released from its inhibitor and move to the nucleus, resulting in transcriptional induction of inflammation-associated genes [58]. Molecular mechanisms, involving the MAPK signaling cascade could control the expression and regulation of several transcription factors. Major serine/threonine protein kinases that constitute MAPK family are kinases like c-jun N-terminal kinase (JNK), extracellular signal-regulated kinase1/2 (Erk1/2) and p38. Several studies showed that fucoidans downregulate NF-KB and MAPK (ERK, JNK, p38) pathways [26,59-62]. Moreover, fucoidan inhibits the transactivation of Activator Protein 1 (AP-1) by attenuating the transcriptional activity of the oncogenes C-Fos or C-Jun. AP-1, in turn, regulates gene expression triggered by stimuli such as cytokines and infections. In this way, fucoidan inhibits pro-inflammatory cytokines production as well as AP-1 response regulated by these mediators [63]. These results were confirmed in a model of myocardial ischemia/reperfusion injury [38]. Fucoidan supplementation decreased the myocardial lesion area, damage, and leukocyte infiltration. Moreover, the blood concentration of inflammatory markers such as TNF, IL-6, and myeloperoxidase activity (neutrophil activity) were reduced while the level of the anti-inflammatory cytokine IL-10 was increased in the fucoidan supplemented group. These findings could be related to lower levels of NF-KB activation, decreased expression of inducible nitric oxide synthase (iNOS) and consequent reduction of nitric oxide production, previously described [28]. A summary of the main biological functions of fucoidan related to antiatherogenic events is shown in Figure 1. Fucoidan is a potential nutraceutical that could be useful as an adjuvant in preventing or treating ACVD

In conclusion, fucoidan has several biological actions that make it a potential adjuvantin preventing or treating ACVD, reducing dyslipidemia, inflammation and thrombus formation. Nonetheless, there are few clinical studies addressed to this subject and future clinical trials are needed to confirm these effects in humans (Figure 1). 


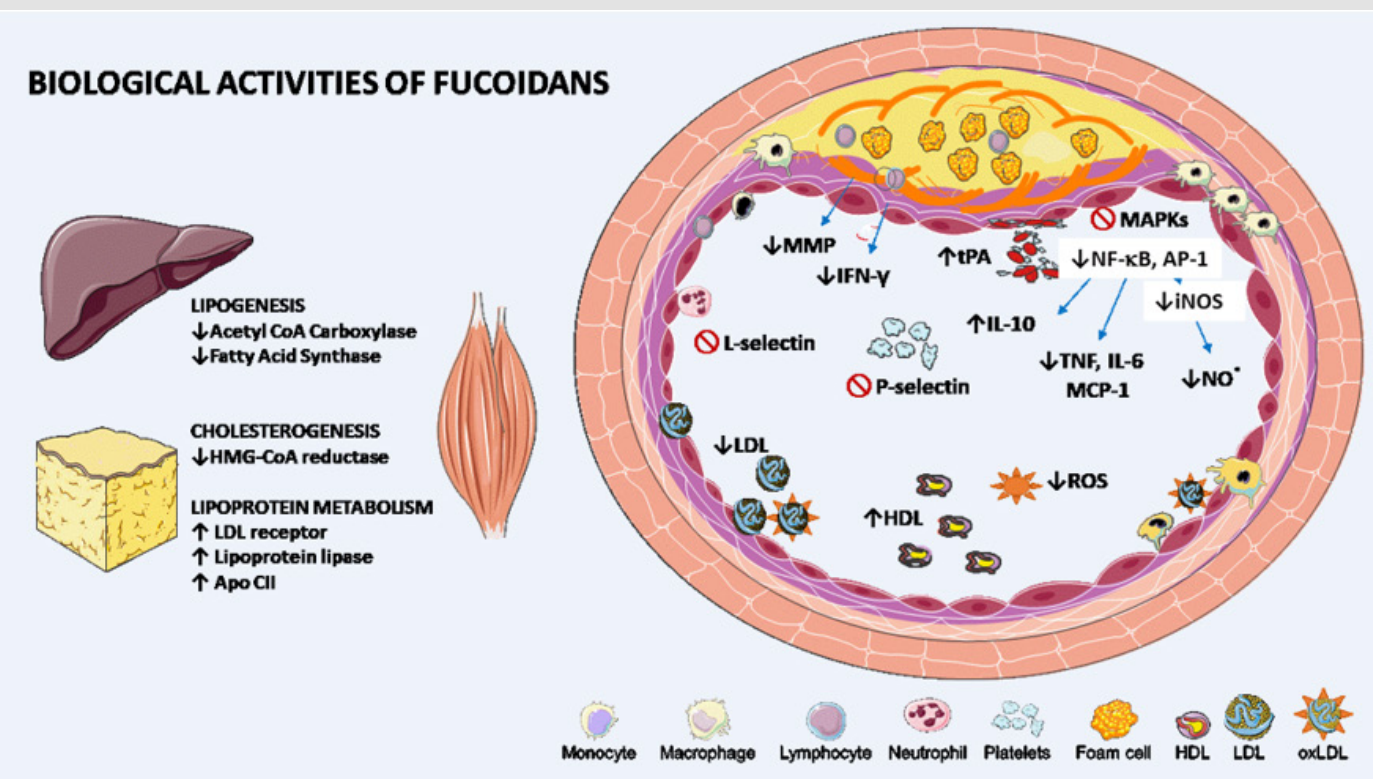

Figure 1: Biological actions of fucoidans that potentially influence atherosclerosis and cardiovascular diseases.

Fucoidan reduces low-density lipoprotein (LDL) by inhibiting the cholesterol synthesis key enzyme hydroxy-methyl-glutaryl CoA (HMG-CoA) reductase and stimulating LDL receptors in the liver and other peripherical tissues. The reduction of fatty acids synthesis associated with the increased lipoprotein lipase (LPL) and its cofactor Apo-CII in adipose tissue leads to lower levels of triglyceride-rich lipoprotein and increase levels of nascent high-density lipoprotein (HDL). Fucoidans also decrease oxidative stress and, consequently, endothelial dysfunction and minimally oxidized LDL formation. Foam cell formation is reduced in the presence of fucoidan due to inhibition of adhesion molecules, which reduces migration of immune cells to the intima layer. Moreover, fucoidan is related with a lower expression of scavenger receptors to ox LDL and lower activation of mitogen-activated protein kinase (MAPK) signaling pathways and transcription factors such as nuclear factor kappa B (NF$\mathrm{KB})$ and activator protein-1 (AP-1). As a result, there is a lower production of inflammatory mediators such as interleukin (IL)-6 and tumor necrosis factor (TNF), monocyte chemotactic protein-1 (MCP1-CCL2) and nitric oxide and the increase of IL-10. The production of matrix metalloproteinases (MMP) by macrophage and interferon-gamma (IFN- $\gamma$ ) by T cells in the presence of fucoidan decreased, reducing fibrous cap degradation and plaque vulnerability. Fucoidan also has anticoagulant and antithrombotic actions by reducing P-selectins (related to platelets aggregation), thrombin and Xa factor and increasing tissue Plasminogen Activator (tPA) and consequently fibrinolysis. iNOS: inducible nitric oxide synthase; NO: Nitric oxide; ROS: reactive oxygen species; $\uparrow:$ increasing; $\downarrow$ : decreasing; $Q$ : inhibition.

\section{Funding}

JIAL is a CNPq (Conselho Nacional de Desenvolvimento Científico e Tecnológico) fellow. ECA and WFB are CAPES (Coordenação de Aperfeiçoamento de Pessoal de Nível Superior) fellows.

\section{Acknowledgement}

None.

\section{Conflict of Interest}

No conflict of interest.

\section{References}

1. Benjamin EJ, Virani SS, Callaway CW, Chamberlain AM, Chang AR, et al (2018) Heart Disease and Stroke Statistics- 2018 Update: A Report from the American Heart Association. Circulation 137(12): e67-e492.

2. Libby P, Buring JE, Badimon L, Hansson GK, Deanfield J, et al. (2019) Atherosclerosis. Nat Rev Dis Prim 5: 56.

3. Buckley ML, Ramji DP (2015) The influence of dysfunctional signaling and lipid homeostasis in mediating the inflammatory responses during atherosclerosis. Biochim Biophys Acta-Mol Basis Dis 1852(7): 14981510
4. Gimbrone MA, García Cardeña G (2016) Endothelial Cell Dysfunction and the Pathobiology of Atherosclerosis. Circ Res 118(4): 620-636.

5. Kavurma MM, Rayner KJ, Karunakaran D (2017) The walking dead: macrophage inflammation and death in atherosclerosis. Curr Opin Lipidol 28(2): 91-98.

6. Ammann KR, De Cook KJ, Tran PL, Merkle VM, Wong PK, et al. (2015) Collective cell migration of smooth muscle and endothelial cells: Impact of injury versus non-injury stimuli. J Biol Eng 9: 1-15.

7. Libby P (2013) Mechanisms of acute coronary syndromes and their implications for therapy. N Engl J Med 368(21): 2004-2013.

8. Mach F, Ray KK, Wiklund O, Corsini A, Catapano AL, et al. (2018) Adverse effects of statin therapy: perception vs. the evidence-focus on glucose homeostasis, cognitive, renal and hepatic function, haemorrhagic stroke and cataract. Eur Heart J 39(27): 2526-2539.

9. Orekhov AN (2018) LDL and foam cell formation as the basis of atherogenesis. Curr Opin Lipidol 29(4): 279-284.

10. Patil NP, Le V, Sligar AD, Mei L, Chavarria D, et al. (2018) Algal Polysaccharides as Therapeutic Agents for Atherosclerosis. Front Cardiovasc Med 5: 153

11.Zaporozhets T, Besednova N (2016) Prospects for the therapeutic application of sulfated polysaccharides of brown algae in diseases of the cardiovascular system: review. Pharm Biol 54(12): 3126-3135. 
12. Li B, Lu F, Wei X, Zhao R (2008) Fucoidan: Structure and bioactivity. Molecules 12-13(8): 1671-1695.

13. Berteau O, Mulloy B (2003) Sulfated fucans, fresh perspectives: Structures, functions, and biological properties of sulfated fucans and an overview of enzymes active toward this class of polysaccharide. Glycobiology 13(6): 29R-40R.

14. Ale MT, Mikkelsen JD, Meyer AS (2011) Important determinants for fucoidan bioactivity: A critical review of structure-function relations and extraction methods for fucose-containing sulfated polysaccharides from brown seaweeds. Mar Drugs 9(10): 2106-2130.

15. Chen K, Febbraio M, Li W, Silverstein RL (2008) A specific cd36dependent signaling pathway is required for platelet activation by oxidized low-density lipoprotein. Circ Res 102(11): 1512-1519.

16. Yokota T, Nagashima M, Ghazizadeh M, Kawanami O (2009) Increased effect of fucoidan on lipoprotein lipase secretion in adipocytes. Life Sci 84(15-16): 523-529.

17. Park J, Yeom M, Hahm DH (2016) Fucoidan improves serum lipid levels and atherosclerosis through hepatic SREBP-2-mediated regulation. J Pharmacol Sci 131(2): 84-92.

18. Huang L, Wen K, Gao X, Liu Y (2010) Hypolipidemic effect of fucoidan from Laminaria japonica in hyperlipidemic rats. Pharm Biol (London, United Kingdom)48(4): 422-426

19. Thomes P, Rajendran M, Pasanban B, Rengasamy R (2010) Cardioprotective activity of Cladosiphon okamuranus fucoidan against isoproterenol induced myocardial infarction in rats. Phytomedicine 18(1): $52-57$

20. Yokota T, Nomura K, Nagashima M, Kamimura N (2016) Fucoidan alleviates high-fat diet-induced dyslipidemia and atherosclerosis in ApoEshl mice deficient in apolipoprotein E expression. J Nutr Biochem 32: 46-54.

21. Omar HEDM, Saad Eldien HM, Badary MS, Al Khatib BY, Abd Elgaffar SK (2013) The immunomodulating and antioxidant activity of fucoidan on the splenic tissue of rats treated with cyclosporine A. J Basic Appl Zool 66(5): 243-254.

22. Rodriguez Jasso R, Mussatto S, Pastrana L, Aguilar C, Teixeira J (2014) Chemical composition and antioxidant activity of sulphated polysaccharides extracted from Fucus vesiculosus using different hydrothermal processes. Chem P 68(2): 203-209.

23. Rocha De Souza MC, Marques CT, Guerra Dore CM, Ferreira Da Silva FR, Oliveira Rocha HA, et al. (2007) Antioxidant activities of sulfated polysaccharides from brown and red seaweeds. J. Appl. Phycol 19(2): 153-160.

24. Xue Z, Changhu X, YUEPIAO C, Dongfeng W, YU F (2005) Study of antioxidant activities of fucoidan from Laminaria japonica. High Technol Lett 11(1): 91-94.

25. Yuan Y, Macquarrie DJ (2015) Microwave assisted extraction of sulfated polysaccharides (fucoidan) from Ascophyllum nodosum and its antioxidant activity. Carbohydr Polym 129: 101-107.

26. Park HY, Han MH, Park C, Jin CY, Kim GY, et al. (2011) Anti-inflammatory effects of fucoidan through inhibition of NF-kB, MAPK and Akt activation in lipopolysaccharide-induced BV2 microglia cells. Food Chem Toxicol 49(8): 1745-1752.

27. Kwak KW, Cho KS, Hahn OJ, Lee KH, Lee BY, et al. (2014) Biological effects of fucoidan isolated from Fucus vesiculosus on thrombosis and vascular cells. Korean J Hematol 45(1): 51-57.

28. Do H, Pyo S, Sohn EH (2010)Suppression of iNOS expression by fucoidan is mediated by regulation of p38 MAPK, JAK/STAT, AP-1 and IRF-1, and depends on up-regulation of scavenger receptor B1 expression in TNF- $\alpha$ and IFN- $\gamma$-stimulated C6 glioma cells 2 . J Nutr Biochem 21(8): 671-679.

29. Lee SH, Ko CI, Jee Y, Jeong Y, Kim M, et al. (2013) Anti-inflammatory effect of fucoidan extracted from Ecklonia cava in zebrafish model. Carbohydr Polym 92(1): 84-89.
30. Sanjeewa KKA, Fernando IPS, Kim SY, Kim HS, Ahn G, et al. (2017) In vitro and in vivo anti-inflammatory activities of high molecular weight sulfated polysaccharide; containing fucose separated from Sargassum horneri: Short communication. Int J Biol Macromol 107(Pt A): 803-807.

31. Kim MJ, Chang UJ, Lee JS (2009) Inhibitory effects of fucoidan in 3T3-L1 adipocyte differentiation. Mar Biotechnol 11(5): 557-562.

32. Kim KJ, Lee OH, Lee BY (2010) Fucoidan, a sulfated polysaccharide, inhibits adipogenesis through the mitogen-activated protein kinase pathway in 3T3-L1 preadipocytes. Life Sci 86(21-22): 791-797.

33. Kim MJ, Jeon J, Lee JS (20140Fucoidan prevents high-fat diet-induced obesity in animals by suppression of fat accumulation. Phyther Res 28(1): 137-143

34. Park MK, Jung U, Roh C (2011) Fucoidan from marine brown algae inhibits lipid accumulation. Mar Drugs 9(8): 1359-1367.

35. Ye C, Wang Y, Su H, Yang P, Huang N, et al. (2016) Construction of a fucoidan/laminin functional multilayer to direction vascular cell fate and promotion hemocompatibility. Mater Sci Eng C 64: 236-242.

36. Drozd NN, Miftakhova NT, Savchik EY, Kalinina TB, Makarov VA, et al. (2011) Antithrombotic and hemorrhagic activities of fucoidan isolated from Fucus evanescens brown algae. Eksp i Klin Farmakol 74(5): 26-30.

37. Camara RBG, Costa LS, Fidelis GP, Nobre LTDB, Dantas Santos N, et al. (2011) Heterofucans from the brown seaweed Canistrocarpus cervicornis with anticoagulant and antioxidant activities. Mar Drugs 9(1): 124-138.

38. Li C, Gao Y, Xing Y, Zhu H, Shen J, et al. (2011) Fucoidan, a sulfated polysaccharide from brown algae, against myocardial ischemiareperfusion injury in rats via regulating the inflammation response. Food Chem Toxicol 49(9): 2090-2095.

39. Krylov VB, Ustyuzhanina NE, Nifantiev NE (2011) Synthesis of lowmolecular-weight carbohydrate mimetics of heparin. Russ J Bioorganic Chem 37(6): 672-706.

40. Wijesinghe WAJP, Jeon YJ (2012) Biological activities and potential industrial applications of fucose rich sulfated polysaccharides and fucoidans isolated from brown seaweeds: A review. Carbohydr Polym 88(1): $13-20$

41. Marinval N, Saboural P, Haddad O, Letourneur D, Charnaux N, et al. (2015) Angiogenesis potentialized by highly sulfated fucoidan: Role of the chemokines and the proteoglycans. Atherosclerosis 241(1): e87.

42. Turnbull J, Powell A, Guimond S (2001) Heparan sulfate: Decoding a dynamic multifunctional cell regulator. Trends Cell Biol 11(2): 75-82.

43. Volpi N (2006) Therapeutic applications of glycosaminoglycans. Curr Med Chem 13(15): 1799-1810.

44. Min SK, Kwon OC, Lee S, Park KH, Kim JK (2011) An antithrombotic fucoidan, unlike heparin, does not prolong bleeding time in a murine arterial thrombosis model: A comparative study of undaria pinnatifida sporophylls and fucus vesiculosus. Phytother Res 26(5): 752-757.

45. Soeda S, Sakaguchi S, Shimeno HNA (1992) Fibrinolytic and anticoagulant activities of highly sulfated fucoidan. Biochem Pharmacol 43(8): 18531858

46. Zhao X, Dong S, Wang J, Li F, Chen A, et al. (2012) A comparative study of antithrombotic and antiplatelet activities of different fucoidans from Laminaria japonica. Thromb Res 129(6): 771-778.

47. Zhang Z, Till S, Jiang C, Knappe S, Reutterer S, et al. (2014) Structureactivity relationship of the pro- and anticoagulant effects of fucus vesiculosus fucoidan. Thromb Haemost 111(3): 429-437.

48. Phull AR, Majid M, Haq Iul, Khan MR, Kim SJ (2017) In vitro and in vivo evaluation of anti-arthritic, antioxidant efficacy of fucoidan from Undaria pinnatifida (Harvey) Suringar. Int J Biol Macromol 97: 468-480.

49. Wang J, Wang F, Zhang Q, Zhang Z, Shi X, et al. (2009) Synthesized different derivatives of low molecular fucoidan extracted from Laminaria japonica and their potential antioxidant activity in vitro. Int 
J Biol Macromol 44(5): 379-384.

50. Morya VK, Kim J, Kim EK (2012) Algal fucoidan: structural and sizedependent bioactivities and their perspectives. Appl Microbiol Biotechnol 93(1): 71-82.

51. Wang X, Pei L, Liu H, Qv K, Xian W, et al. (2016) Mice Through Inhibition of Inflammation and Oxidative Stress. Int J Clin Exp Pathol 9: 6896-6904.

52. Liang Z, Zheng Y, Wang J, Zhang Q Ren S, et al. (2016). Low molecular weight fucoidan ameliorates streptozotocin-induced hyperresponsiveness of aortic smooth muscles in type 1 diabetes rats. J Ethnopharmacol 191: 341-349

53. Kim KJ, Yoon KY, Lee BY (2012) Low molecular weight fucoidan from the sporophyll of Undaria pinnatifida suppresses inflammation by promoting the inhibition of mitogen-activated protein kinases and oxidative stress in RAW264.7 cells. Fitoterapia 83(8): 1628-1635.

54. Ryu MJ, Chung HS (2016) Fucoidan reduces oxidative stress by regulating the gene expression of H0-1 and SOD-1 through the Nrf2/ERK signaling pathway in HaCaT cells. Mol Med Rep 14(4): 3255-3260.

55. Simonis D, Fritzsche J, Alban S, Bendas G (2007) Kinetic analysis of heparin and glucan sulfates binding to P-selectin and its impact on the general understanding of selectin inhibition. Biochemistry 46(20): 6156-6164.

56. Fitton JH (2011) Therapies from fucoidan; multifunctional marine polymers. Mar Drugs 9(10): 1731-1760.

\section{ISSN: 2574-1241}

DOI: 10.26717/BJSTR.2019.21.003616

Weslley F Braga. Biomed J Sci \& Tech Res

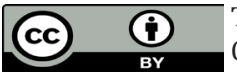

This work is licensed under Creative Commons Attribution 4.0 License

Submission Link: https://biomedres.us/submit-manuscript.php
57. Woollard KJ, Chin Dusting J (2007) Therapeutic targeting of p-selectin in atherosclerosis. Inflamm Allergy Drug Targets 6(1): 69-74.

58. Zhang Q, Lenardo MJ, Baltimore D (2016) 30 Years of NF-kB: A Blossoming of Relevance to Human Pathobiology. Cell 168(1-2): 37-57.

59. Patel MK, Mulloy B, Gallagher KL, O’Brien L, Hughes AD (2002) The antimitogenic action of the sulphated polysaccharide fucoidan differs from heparin in human vascular smooth muscle cells. Thromb Haemost 87(1): 149-154.

60. Sanjeewa KKA, Fernando IPS, Kim EA, Ahn G, Jee Y, et al. (2017) Antiinflammatory activity of a sulfated polysaccharide isolated from an enzymatic digest of brown seaweed Sargassum horneri in RAW 264.7 cells. Nutr Res Pract 11(1): 3-10.

61. Che N, Ma Y, Xin Y (2017) Protective role of fucoidan in cerebral ischemia-reperfusion injury through inhibition of MAPK signaling pathway. Biomol Ther 25(3): 272-278.

62. Choo GS, Lee HN, Shin SA, Kim HJ, Jung JY (2016) Anticancer effect of fucoidan on DU-145 prostate cancer cells through inhibition of PI3K/ Akt and MAPK pathway 17 expression. Mar Drugs 14(7).

63. Lee NY, Ermakova SP, Zvyagintseva TN, Kang KW, Dong Z, et al. (2008) Inhibitory effects of fucoidan on activation of epidermal growth factor receptor and cell transformation in JB6 Cl41 cells. Food Chem Toxicol 46(5): 1793-1800.

BIOMEDICAL
RESEARCHES $\quad$\begin{tabular}{l} 
Assets of Publishing with us \\
\hline - Immediate, unrestricted online access
\end{tabular}

